\title{
Authors Response: Letter to Editor: Lower Urinary Tract Mesh Perforations Following the Insertion of Mid-Urethral Tapes or Transvaginal Mesh: A Tertiary Unit's Experience - Saidan, et al. 2019
}

\author{
Dalia Saidan $^{1 *}$, Veenu Tyagi ${ }^{1}$, Paraskeve (Voula) Granitsiotis ${ }^{2}$ and Karen Guerrero ${ }^{1}$ \\ ${ }^{1}$ Queen Elizabeth University Hospital, Glasgow, United Kingdom \\ ${ }^{2}$ Western Infirmary, Edinburgh, United Kingdom
}

*Corresponding author: Dalia Saidan, Queen Elizabeth University Hospital, 1345 Govan Road, Glasgow G51 4TF, United Kingdom

\section{Dear Editor-in-Chief,}

It has come to our attention that a letter has been published [1] following publication of our article, Lower Urinary Tract Mesh Perforations Following the Insertion of Mid-urethral Tapes or Transvaginal Mesh: A Tertiary Unit's Experience - Saidan, et al. 2019 [2], by one of our colleagues, and we would be grateful for an opportunity to reply.

Our published paper [2] relates to a small retrospective case-series of women undergoing surgery for management of their urinary tract mesh perforation only. It therefore has its limitations, which we have acknowledged in our paper. We are grateful to the Editor, and the scientific team who reviewed our paper as part of the peer-reviewed process, for con- sidering it worthy of publication despite these.

We thank Dr. Agur [1] for his helpful suggestions, which we will take into consideration for any future publications of our work. Many of the questions he raises however do not relate to this case series and are outside the remit of this paper. We can however clarify the following points that do relate to this case series.

The 8 patients highlighted by Dr. Agur presented with symptoms to their local medical teams within 8-weeks of mesh insertion (Table 1). This was not when they were referred to or seen in our tertiary unit, or had their surgery.

We have acknowledged in the paper that some of our patients who underwent excision of mesh to man-

Table 1: Time from implant insertion to onset of symptoms.

\begin{tabular}{|l|l|l|}
\hline $\begin{array}{l}\text { Time from Implant insertion to symptom } \\
\text { development }\end{array}$ & IUGA Classification & Number of patients (\%) \\
\hline$<48$ hours & T1 & 0 \\
\hline$<2$ months & T2 & $8(20 \%)$ \\
\hline 2-12 months & T3 & $10(25 \%)$ \\
\hline 1-3 years & T4 & $5(12.5 \%)$ \\
\hline 3-5 years & T4 & $5(12.5 \%)$ \\
\hline 5+ years & T4 & $12(30 \%)$ \\
\hline
\end{tabular}

Citation: Saidan D, Tyagi V, Paraskeve (Voula) P, Guerrero K (2020) Authors Response: Letter to Editor: Lower Urinary Tract Mesh Perforations Following the Insertion of Mid-Urethral Tapes or Transvaginal Mesh: A Tertiary Unit's Experience - Saidan, et al. 2019. Int Arch Urol Complic 6:070. doi. org/10.23937/2469-5742/1510070

Accepted: April 06, 2020: Published: April 08, 2020

Copyright: (C) 2020 Saidan D, et al. This is an open-access article distributed under the terms of the Creative Commons Attribution License, which permits unrestricted use, distribution, and reproduction in any medium, provided the original author and source are credited. 
age their perforation were subsequently found to have some residual some mesh fibres in the bladder. These were treated cystoscopically with symptom resolution. We have already commented on our learning curve as a unit in the discussion, with some of our historic cases being included in this case series. It is important that clinicians learn from these early experiences of clinicians performing this work.

We are also of the opinion, as Dr. Agur states in his letter, that it is likely that presentations within 12 months of mesh placement are potentially missed intraoperative injuries. We have stated so in our discussion.

We welcome the recent publications by the American Urogynecologic Society and the International Urogynecological Association International [3]. It provides guidance to subspecialists in Female Pelvic Medicine and Re-constructive Surgery (FPMRS) on assessment, investigation and management of women presenting with potential mesh complications, and also their efforts to categorize these surgical procedures and their nomenclature.
In line with national UK guidelines, we continue to upload our mesh- removal data onto the British Society of Urogynaecology database [4].

We are glad our paper has generated debate and interest. We encourage other FPMRS Sub-specialists to share their experiences. It is important that we all continue to learn from others in this emerging field.

\section{References}

1. Wael Agur (2019) Letter to Editor: Lower urinary tract mesh perforations following the insertion of mid-urethral tapes or transvaginal mesh: A tertiary unit's experience - Saidan, et al. 2019. Int Arch Urol Complic 5: 1-2.

2. Saidan D, Tyagi V, Granitsiotis P, Guerrero K (2019) Lower urinary tract mesh perforations following insertion of mid-urethral tapes or transvaginal mesh: A tertiary unit's experience. Int Arch Urol Complic 5: 1-6.

3. Joint position statement on the management of mesh-related complications for the FPMRS specialist. Developed by the Joint Writing Group of the American Urogynecologic Society and the International Urogynecological Association. Int Urogynecol J.

4. BSUG audit database. 